\title{
REIDEMEISTER TORSION AND INTEGRABLE HAMILTONIAN SYSTEMS
}

\section{ALEXANDER FEL'SHTYN and HECTOR SÁNCHEZ-MORGADO}

\author{
(Received 7 December 1998)
}

\begin{abstract}
In this paper, we compute the Reidemeister torsion of an isoenergetic surface for the integrable Hamiltonian system on the 4-dimensional symplectic manifold. We use the spectral sequence defined by the filtration and following Floer-Witten ideas we bring into play the orbits connecting the critical submanifolds.
\end{abstract}

Keywords and phrases. The Reidemeister torsion, integrable Hamiltonian systems, isoenergetic surface, symplectic manifold, Bott integral, critical circles.

1991 Mathematics Subject Classification. Primary 58F; Secondary 57Q10.

1. Introduction. Reidemeister torsion is a very important topological invariant which has useful applications in knot theory, quantum field theory and dynamical systems. In 1935, Reidemeister [10] classified up to PL equivalence the lens spaces $S^{3} / \Gamma$, where $\Gamma$ is a finite cyclic group of fixed point free orthogonal transformations. He used a certain new invariant-the Reidemeister torsion-which was quickly extended by Franz, who used it to classify the generalized lens spaces $S^{2 n+1} / \Gamma$. Let $X$ be a compact smooth manifold. A representation $\rho: \pi_{1}(X) \rightarrow U(m)$ of the fundamental group defines a flat $\mathbb{C}^{m}$ bundle $E$ over $X$. When the twisted cohomology $H^{*}(X ; E)$ vanishes, the representation $\rho$ and the flat bundle $E$ are called acyclic. The Reidemeister torsion or $R$-torsion is a positive number which is a ratio of determinants concocted from the $\pi_{1}(X)$-equivariant chain complex of the universal covering of $X$. Later, Milnor identified the Reidemeister torsion with the Alexander polynomial, which plays a fundamental role in the theory of knots and links.

In 1971, Ray and Singer [9] introduced an analytic torsion associated with the de Rham complex of forms with coefficients in a flat bundle over a compact Riemannian manifold, and conjectured it was the same as the Reidemeister torsion. The Ray-Singer conjecture was established independently by Cheeger and Müller a few years later.

Recently, the Reidemeister torsion has found interesting applications in dynamical systems theory. A connection between the Lefschetz type dynamical zeta functions and the Reidemeister torsion was established by D. Fried [6]. The work of Milnor [8] was the first indication that such a connection exists.

In this paper, we study the Reidemeister torsion of isoenergy surfaces of an integrable Hamiltonian system. Let $N$ be a 4-dimensional smooth symplectic manifold and consider the Hamiltonian system with smooth Hamiltonian $H$, which in Darboux coordinates has the form 


$$
\frac{d p_{i}}{d t}=-\frac{\partial H}{\partial q_{i}}, \quad \frac{d q_{i}}{d t}=\frac{\partial H}{\partial p_{i}} .
$$

The 3-dimensional level surface $M=\{H=$ constant $\}$ is invariant under the flow defined by the system (1.1). The surface $M$ is called an isoenergy surface or a constantenergy surface. The topological structure of isoenergy surfaces of integrable Hamiltonian systems and the structure of their fundamental groups were described in $[3,4]$. Isoenergy surfaces of integrable Hamiltonians system possess specific properties which distinguish them among all smooth 3-dimensional manifolds. Namely, they belong to the class of graph-manifolds introduced by Waldhausen [12]. Since $N$ is orientable (as a symplectic manifold), the surface $M$ is automatically orientable in all cases. Suppose that the system (1.1) is complete integrable (in Liouville's sense) on the surface $M$. This means, that there is a smooth function $f$ (the second integral), which is independent of $H$ and with Poisson bracket $\{H, f\}=0$ in a neighborhood of $M$.

Definition. We call $f: M \rightarrow \mathbb{R}$ a Bott function if its critical points form critical nondegenerate smooth submanifolds of $M$. This means that the Hessian $d^{2} f$ of the function $f$ is nondegenerate on the planes normal to the critical submanifolds of the function $f$.

Fomenko [3] proved that a Bott integral on a compact nonsingular isoenergy surface $M$ can have only three types of critical submanifolds: circles, tori, or Klein bottles. The investigation of concrete mechanical and physical systems [3] shows that it is a typical situation when the integral on $M$ is a Bott integral. In the classical integrable cases of the solid body motion (cases of the Kovalevskaya, Goriachev-Chaplygin, Clebsch, Manakov) the Bott integrals are round Morse functions on the isoenergy surfaces. A round Morse function is a Bott function all whose critical manifolds are circles. Note that critical circles of $f$ are periodic solutions of the system (1.1) and the number of these circles is finite. Suppose for the moment that the Bott integral $f$ is a round Morse function on the closed isoenergy surface $M$. Let us recall the concept of the separatrix diagram of the critical circle $\gamma$. Let $x \in \gamma$ be an arbitrary point and $N_{x}(\gamma)$ be a disc of small radius normal to $\gamma$ at $x$. The restriction of $f$ to the $N_{x}(\gamma)$ is a normal Morse function with the critical point $x$ having a certain index $u(\gamma)=0,1,2$. A separatrix of the critical point $x$ is an integral trajectory of the field $\operatorname{grad} f$, called a gradient line, which is entering or leaving $x$. The union of all the separatrices leaving the point $x$ gives a disc of dimension $u(\gamma)$ and is called the outgoing separatrix diagram (disc). The union of incoming separatrices gives a disc of complementary dimension and is called the separatrix incoming diagram (disc). Varying the point $x$ and constructing the incoming and outgoing separatrix discs for each point $x$, we obtain the incoming and outgoing separatrix diagrams of the circle $\gamma$. Let $\Delta(\gamma)$ be +1 if the outgoing separatrix is orientable, and -1 if it is not. Let $\epsilon(\gamma)=(-1)^{u(\gamma)}$. Let $\rho_{E}: \pi_{1}(M, p) \rightarrow U\left(E_{p}\right)$ be the holonomy representation of the hermitian bundle $E$ over $M$; $E_{p}$ is the fiber at the point $p$. For the gradient flow of $f$ on $M$, one can construct an index filtration for the collection of critical circles $\left\{\gamma_{i}\right\}$, i.e., a collection of compact submanifolds $M_{i}$ of top dimension so that $M_{i} \subset$ int $M_{i+1}, M_{0}=\emptyset, M_{i}=M$ for large $i$, the flow is transverse inwards on $\partial M_{i}$ and $M_{i+1} \backslash M_{i}$ is an isolating neighborhood for the critical circle $\gamma_{i}$. 
When $E \mid\left(M_{i+1}, M_{i}\right)$ is acyclic for each $i$, following the ideas of D. Fried [6] we can compute the Reidemeister torsion as [1]

$$
\tau(M ; E)=\prod_{i} \tau\left(M_{i+1}, M_{i} ; E\right)=\prod_{\gamma_{i}}\left|\operatorname{det}\left(I-\Delta\left(\gamma_{i}\right) \cdot \rho_{E}\left(\gamma_{i}\right)\right)\right|^{\varepsilon\left(\gamma_{i}\right)} .
$$

This formula means that for the integrable Hamiltonian system on the 4-dimensional symplectic manifold, the Reidemeister torsion of the isoenergy surface counts the critical circles of the second independent Bott integral on this surface. If $E \mid\left(M_{i+1}, M_{i}\right)$ is acyclic, then $\operatorname{det}\left(I-\Delta\left(\gamma_{i}\right) \cdot \rho_{E}\left(\gamma_{i}\right)\right) \neq 0$ for each $i$. Since in many classical integrable cases there are contractible critical circles it is interesting to study the situation when not all $E \mid\left(M_{i+1}, M_{i}\right)$ are acyclic. Here, we carry out this study and in fact we consider the general situation when the Bott integral has critical tori and Klein bottles. We use the spectral sequence defined by the filtration and following Witten-Floer ideas we bring into play the orbits connecting the critical submanifolds. A similar approach was developed in [11] for Morse-Smale flows.

2. $R$-torsion and spectral sequences. Let $W$ be a finite dimensional vector space with basis $\mathbf{w}=\left\{w_{1}, \ldots, w_{n}\right\}$, then $\wedge \mathbf{w}=w_{1} \wedge \cdots \wedge w_{n}$ is a generator of $\operatorname{det} W=\wedge^{n} W$. If $\operatorname{dim} V=0$, set det $V=\mathbb{C}$.

Consider a cochain complex of finite dimensional vector spaces

$$
0 \longrightarrow V^{0} \stackrel{d}{\longrightarrow} V^{1} \longrightarrow \cdots \longrightarrow V^{m} \stackrel{d}{\longrightarrow} 0 .
$$

Let $V^{+}=\bigoplus_{i} V^{2 i}, V^{-}=\bigoplus_{i} V^{2 i+1}$, and

$$
\operatorname{det} V=\operatorname{det}\left(V^{-}\right) \otimes\left(\operatorname{det} V^{+}\right)^{-1} .
$$

Let $Z^{ \pm}=V^{ \pm} \cap \operatorname{ker} d, B^{\mp}=d\left(V^{ \pm}\right), H^{ \pm}=Z^{ \pm} / B^{ \pm}$.

We now define the torsion element $\tau_{d} \in \operatorname{det} V \otimes(\operatorname{det} H)^{-1}$. Pick ordered relative bases $\mathbf{h}_{ \pm}$for $\left(Z^{ \pm}, B^{ \pm}\right)$and $\mathbf{t}_{ \pm}$for $\left(V_{ \pm}, Z_{ \pm}\right)$, then $d \mathbf{t}_{\mp}$ is a basis for $B_{ \pm}$. Denote by $\left[\mathbf{h}_{ \pm}\right]$the corresponding basis for $H^{ \pm}$.

$$
\tau_{d}=\wedge\left(\mathbf{t}_{-}, \mathbf{h}_{-}, d \mathbf{t}_{+}\right) \otimes \wedge\left(d \mathbf{t}_{-}, \mathbf{h}_{+}, \mathbf{t}_{+}\right)^{-1} \otimes \wedge\left[\mathbf{h}_{+}\right] \otimes \wedge\left[\mathbf{h}_{-}\right]^{-1} .
$$

Notation. Consider the cochain complex

$$
0 \longrightarrow V \stackrel{A}{\longrightarrow} W \longrightarrow 0 .
$$

We have $H^{0}=\operatorname{ker} A, H^{1}=\operatorname{coker} A$ and denote $\tau(A):=\tau_{d}$. When $A$ is an isomorphism, $\tau(A)$ is the coordinate free version of $\operatorname{det} A$.

Proposition 1 [5]. Let $0=F_{N+1}^{i} \subset F_{N}^{i} \subset \cdots \subset F_{0}^{i}=V^{i}$ be a filtration of the cochain complex (2.1) such that $d^{i}\left(F_{n}^{i}\right) \subset F_{n}^{i+1}$. Let $\left\{E_{r}, d_{r}\right\}$ be the corresponding spectral sequence. Then

$$
\tau_{d}=\tau_{d_{0}} \otimes \cdots \otimes \tau_{d_{N}}
$$


COROLlary 2 [5]. Suppose

$$
0 \longrightarrow\left(C^{\prime}, d^{\prime}\right) \stackrel{i}{\longrightarrow}(C, d) \stackrel{j}{\longrightarrow}\left(C^{\prime \prime}, d^{\prime \prime}\right) \longrightarrow 0
$$

is an exact sequence of chain complexes and

$$
\mathscr{H}: 0 \longrightarrow H^{0}\left(C^{\prime}\right) \stackrel{i^{*}}{\longrightarrow} H^{0}(C) \longrightarrow H^{0}\left(C^{\prime \prime}\right) \stackrel{\partial}{\longrightarrow} H^{1}\left(C^{\prime}\right) \longrightarrow \cdots
$$

is the corresponding long exact sequence. For each $k$ choose compatible volume elements in $\operatorname{det} C_{k}^{\prime}$, $\operatorname{det} C_{k}$, $\operatorname{det} C_{k}^{\prime \prime}$, i.e., such that the torsion of (2.6) is 1 . Then

$$
\tau_{d}=\tau_{d^{\prime}} \tau_{d^{\prime \prime}} \tau_{\mathscr{H}}
$$

We now describe the first terms of the spectral sequence $\left\{E_{r}, d_{r}\right\}$. The filtration defines the associated graded complex $G^{i}=\bigoplus_{n} G_{n}^{i}$ where $G_{n}^{i}=F_{n}^{i} / F_{n+1}^{i}$. The coboundary $d$ induces a map $d_{0}^{n}: G_{n}^{i} \rightarrow G_{n}^{i+1}$ whose cohomology defines the term $E_{1}$ by

$$
E_{1}^{n, q}:=H^{n+q}\left(\frac{F_{n}}{F_{n+1}}\right),
$$

and induces the first differential $d_{1}^{n}: E_{1}^{n, q} \rightarrow E_{1}^{n+1, q}$ as the coboundary map for the short exact sequence

$$
0 \longrightarrow \frac{F_{n+1}}{F_{n+2}} \longrightarrow \frac{F_{n}}{F_{n+2}} \longrightarrow \frac{F_{n}}{F_{n+1}} \longrightarrow 0
$$

i.e., the map $d_{1}$ in the long exact sequence

$$
\stackrel{j_{n}}{\longrightarrow} H^{n+q}\left(\frac{F_{n}}{F_{n+2}}\right) \stackrel{k_{n}}{\longrightarrow} H^{n+q}\left(\frac{F_{n}}{F_{n+1}}\right) \stackrel{d_{1}}{\longrightarrow} H^{n+q+1}\left(\frac{F_{n+1}}{F_{n+2}}\right) .
$$

The term $E_{2}^{n, q}$ is defined by

$$
E_{2}^{n, q}:=\frac{\operatorname{ker}\left(d_{1}: E_{1}^{n, q} \longrightarrow E_{1}^{n+1, q}\right)}{\operatorname{im}\left(d_{1}: E_{1}^{n-1, q} \longrightarrow E_{1}^{n, q}\right)} .
$$

From (2.11) we have $\operatorname{ker}\left(d_{1}\right)=\operatorname{im}\left(k_{n}\right)$ and $\operatorname{im}\left(d_{1}\right)=\operatorname{ker}\left(j_{n-1}\right)$, and thus $E_{2}^{n, q}=$ $\operatorname{im}\left(k_{n}\right) / \operatorname{ker}\left(j_{n-1}\right)$.

Consider the commutative diagram

$$
\begin{aligned}
& H^{n+q}\left(\frac{F_{n}}{F_{n+2}}\right) \underset{\delta_{0}}{\stackrel{k_{n}}{\longrightarrow}} H^{n+q}\left(\frac{F_{n}}{F_{n+1}}\right) \underset{\delta_{1}}{\stackrel{j_{n-1}}{\longrightarrow}} H^{n+q}\left(\frac{F_{n-1}}{F_{n+1}}\right) \\
& H^{n+q-1}\left(\frac{F_{n-2}}{F_{n}}\right) \stackrel{k_{n-2}}{\longrightarrow} H^{n+q-1}\left(\frac{F_{n-2}}{F_{n-1}}\right) \stackrel{j_{n-3}}{\longrightarrow} H^{n+q-1}\left(\frac{F_{n-3}}{F_{n-1}}\right) \text {. }
\end{aligned}
$$

The second differential $d_{2}^{n-2}: E_{2}^{n-2, q+1} \rightarrow E_{2}^{n, q}$ is given as the composite map

$$
\frac{\operatorname{im}\left(k_{n-2}\right)}{\operatorname{ker}\left(j_{n-3}\right)} \stackrel{\delta_{1}}{\longrightarrow} \operatorname{im}\left(j_{n-1}\right) \stackrel{j_{n-1}^{-1}}{\longrightarrow} \frac{\operatorname{im}\left(k_{n}\right)}{\operatorname{ker}\left(j_{n-1}\right)} \text {. }
$$


Further terms of the spectral sequence $E_{r}^{n, q}$ are obtained as cohomology of the previous term and the differentials $d_{r}^{n}: E_{r}^{n, q} \rightarrow E_{r}^{n+r, q+r-1}$ are the maps induced by the original $d$.

Now, let $K$ be a finite CW-complex. Let $p: \tilde{K} \rightarrow K$ be the universal covering and $\rho: \Gamma \rightarrow U(m)$ be a representation of the fundamental group $\Gamma$ of $K$ which defines a flat vector bundle $E:=\tilde{K} \times_{\Gamma} \mathbb{C}^{m}$. Lifting cells to $\tilde{K}$ we obtain a $\Gamma$-invariant $\mathrm{CW}$-complex structure on $\tilde{K}$. The space of $\rho$-equivariant cochains

$$
C^{*}(K ; E)=\left\{\xi \in C^{*}\left(\tilde{K} ; \mathbb{C}^{m}\right): \xi \circ \gamma=\rho(\gamma) \circ \xi \forall \gamma \in \Gamma\right\}
$$

is preserved by $d^{j}: C^{j}\left(\tilde{K} ; \mathbb{C}^{m}\right) \rightarrow C^{j+1}\left(\tilde{K} ; \mathbb{C}^{m}\right)$ and so $\left\{C^{*}(K ; E), d(K ; E)\right\}$ forms a subcomplex. Its cohomology $H^{*}(K ; E)$ is called the $\rho$-twisted cohomology of $K$. As usual $H^{*}(K ; E)$ is subdivision invariant and we have a torsion element

$$
\tau_{d(K ; E)} \in \operatorname{det} C^{*}(K ; E) \otimes\left(\operatorname{det} H^{*}(K ; E)\right)^{-1} .
$$

Order the $j$-cells $\sigma$ and choose an oriented lift $\tilde{\sigma}$ for each $\sigma$. This gives an isomorphism $C^{j}(K ; E) \cong \oplus_{\sigma} \mathbb{C}^{m}$ and determines a preferred generator $w_{K}^{\rho}$ of $\operatorname{det}\left(C^{*}(K ; E)\right)$ up to multiplication by an element of the subgroup

$$
U_{\rho}=\left\{( \pm 1)^{m} \operatorname{det} \rho(\gamma): \gamma \in \Gamma\right\} \subset S^{1} .
$$

The orbit $U_{\rho} w_{K}^{\rho} \subset \operatorname{det} C^{*}(K ; E)$ is invariant under subdivision, so we can define $R$-torsion of $K$ at $\rho$ as the $U_{\rho}$ orbit

$$
\tau(K ; E)=\left(U_{\rho} w_{K}^{\rho}\right)^{-1} \otimes \tau_{d(K ; E)} \subset\left(\operatorname{det} H^{*}(K ; E)\right)^{-1},
$$

which is invariant under subdivision. When $\rho$ is acyclic, i.e., when $H^{*}(K ; E)=0$, we have $\operatorname{det} H^{*}(K ; E)=\mathbb{C}$ and we can identify $\tau(K ; E)$ as an element of $\mathbb{C}^{*} / U_{\rho}$. Since $U_{\rho} \subset S^{1}$, all elements in $\tau(K ; E)$ have the same modulus which we still denote by $\tau(K ; E)$.

The previous definitions can be extended to relative pairs. Let $L$ be a subcomplex of $K$. For each $j$ we have the relative space of cellular $j$-cochains

$$
C^{j}(K, L ; \mathbb{C})=\bigoplus_{\sigma \in K \backslash L} H^{j}(\sigma, \partial \sigma ; \mathbb{C}) .
$$

Let $\tilde{K}$ and $\rho$ be as above and let $\tilde{L}=p^{-1}(L)$. We can define the space of relative $\rho$-equivariant cochains $C^{*}(K, L ; E) \subset C^{*}\left(\tilde{K}, \tilde{L} ; \mathbb{C}^{m}\right)$ with coboundary $d(K, L ; E)$ and then we get a torsion element

$$
\tau_{d(K, L ; E)} \in \operatorname{det} C^{*}(K, L ; E) \otimes\left(\operatorname{det} H^{*}(K, L ; E)\right)^{-1} .
$$

Thus, choosing preferred basis as before we obtain a $U_{\rho}$ orbit

$$
\tau(K, L ; E) \subset \operatorname{det} H^{*}(K, L ; E)^{-1},
$$

which is invariant under subdivision.

REMARK 1. Another name for the twisted cohomology is cohomology with local coefficients. One chooses a point on each cell of $K$ and a path from a fixed point to each chosen point. In this way any path $c$ between chosen points defines a closed path 
$\gamma_{c}$ and then a matrix $\rho(c):=\rho\left(\gamma_{c}\right)$ which gives the relation between the coefficients at the ends of the path.

3. $R$-torsion and critical submanifolds. The foliation of the isoenergy surface $M$ by Liouville tori is given by the level sets of the Bott integral $f^{-1}(c)$ for $c$ a regular value. The bifurcation of Liouville tori occur at the sets $F_{c}=f^{-1}(c) \cap$ Crit $(f)$ for $c$ a critical value. We will make the following assumption which is satisfied in the generic case.

Assumption. We will assume that there are no gradient lines of the Bott integral $f$ connecting saddle circles, i.e., circles with index 1 .

We will substitute the Bott integral for another Bott function, still denoted by $f$ and not necessarily an integral, giving the same foliation by Liouville tori and such that its critical values $c_{1}<\cdots<c_{l}$, are ordered in the following way

(a) $i \leq k_{1} \Leftrightarrow F_{c_{i}}$ is a minimum circle.

(b) $k_{1}<i \leq k_{2} \Leftrightarrow F_{c_{i}}$ is a minimum torus or Klein bottle.

(c) $k_{3}<i \leq k_{4} \Leftrightarrow F_{c_{i}}$ is a maximum torus or Klein bottle.

(d) $k_{4}<i \Leftrightarrow F_{c_{i}}$ is a maximum circle.

Choosing numbers $A_{0}<c_{1}<A_{1}<\cdots<c_{l}<A_{l}$ and letting $N_{j}=f^{-1}\left(\infty, A_{j}\right]$ we get an index filtration $\emptyset=N_{0} \subset N_{1} \subset \cdots \subset N_{l}=M$ for the critical sets of $f$, i.e., denoting by $\phi$ the gradient flow of $f, \phi$ is transverse inwards on $\partial N_{n}$ and

$$
\bigcap_{t \in \mathbb{R}} \phi_{t}\left(N_{j} \backslash N_{j-1}\right)=F_{c_{j}} .
$$

Fix a representation $\rho: \pi_{1}(M) \rightarrow U(m)$. All cochain complexes and cohomology groups will have coefficients in the flat bundle defined by $\rho$. Let $l_{0}=0, l_{1}=k_{2}, l_{2}=k_{3}, l_{3}=l$ and $M_{n}=N_{l_{n}}$. Define the filtration $F_{5} \subset \cdots \subset F_{1} \subset F_{0}$ of $C^{*}(M)$ by $F_{n}=\operatorname{ker}\left(C^{*}(M) \rightarrow\right.$ $\left.C^{*}\left(M_{n}\right)\right)$. The associated graded complex is given by

$$
G_{n}=\frac{F_{n}}{F_{n+1}} \cong C^{*}\left(M_{n+1}, M_{n}\right)
$$

with 0-differential $d_{0}=\bigoplus_{n} d_{0}^{n}$, where $d_{0}^{n}: G_{n} \rightarrow G_{n}$, and torsion element $\tau_{d_{0}}=\bigotimes_{n} \tau_{d_{0}^{n}}$, where

$$
\tau_{d_{0}^{n}} \in \operatorname{det} C^{*}\left(M_{n+1}, M_{n}\right) \otimes\left(\operatorname{det} H^{*}\left(M_{n+1}, M_{n}\right)\right)^{-1} .
$$

Since there are neither gradient lines connecting two minimum (maximum) critical submanifolds nor gradient lines connecting two saddle circles (by assumption), we have (see [11])

$$
\begin{gathered}
H^{*}\left(M_{n+1}, M_{n}\right)=\bigoplus_{M_{n} \subset N_{j} \subset M_{n+1}} H^{*}\left(N_{j}, N_{j-1}\right), \\
\tau_{d_{0}^{n}}=\bigotimes_{M_{n} \subset N_{j} \subset M_{n+1}} \tau_{d_{0}^{n, j}} .
\end{gathered}
$$

From (3.4), the computation of the map

$$
d_{1}^{n}: H^{*}\left(M_{n}, M_{n-1}\right) \rightarrow H^{*+1}\left(M_{n+1}, M_{n}\right)
$$


reduces to computing for each $i, j$ with $l_{n-1}<i \leq l_{n}<j \leq l_{n+1}$, its component $F_{i j}^{*}: H^{*}\left(N_{i}, N_{i-1}\right) \rightarrow H^{*+1}\left(N_{j}, N_{j-1}\right)$. To do so, we will use the trajectories of the gradient flow of $f$, but we will modify $f$ in the neighborhood of each critical level set in order to apply Lemma 1 below giving such a map in the Morse function case. This modification is just a technical device to choose some of the orbits connecting the critical submanifolds to describe the maps $F_{i j}^{*}$. We will give a proof of the following proposition using Lemma 1.

Proposition 3. If $k_{2 n}<j \leq k_{2 n+1}$, let $\gamma_{j}=F_{c_{j}}$ and $\mathscr{D}_{j}=I-\Delta\left(\gamma_{j}\right) \rho\left(\gamma_{j}\right)$. Then

$$
\begin{gathered}
H^{k}\left(N_{j}, N_{j-1}\right)= \begin{cases}\operatorname{ker} \mathscr{D}_{j}, & \text { if } k=n, n+1, \\
0, & \text { in other case, }\end{cases} \\
\tau_{d_{0}^{n, j}}=\tau\left(\mathscr{D}_{j}\right)^{(-1)^{n}} .
\end{gathered}
$$

If $k_{2 n-1}<j \leq k_{2 n}$ and $\alpha_{j}, \beta_{j}$ are generators of the fundamental group of $F_{c_{j}}$, let $\mathscr{D}_{j}=\left(\begin{array}{c}I-\rho\left(\alpha_{j}\right) \\ I \pm \rho\left(\beta_{j}\right)\end{array}\right), \mathscr{D}_{j}^{*}=\left(I \pm \rho\left(\beta_{j}\right), \rho\left(\alpha_{j}\right)-I\right)$, where the + sign occurs precisely when $F_{c_{j}}$ is a Klein bottle. Then

$$
\begin{gathered}
H^{k}\left(N_{j}, N_{j-1}\right)= \begin{cases}\operatorname{ker} \mathscr{D}_{j}=\text { coker } \mathscr{D}_{j}^{*}, & \text { if }|k-n|=1, \\
\operatorname{ker} \mathscr{D}_{j} \oplus \operatorname{ker} \mathscr{D}_{j}, & \text { if } k=n, \\
0, & \text { in other case, }\end{cases} \\
\qquad \tau_{d_{0}^{n-1, j}}=1 .
\end{gathered}
$$

\section{COROLlaRY 4.}

$$
\begin{aligned}
& E_{1}^{0, q}=H^{q}\left(M_{1}\right)=0 \quad \text { for } q \neq 0,1,2, \\
& E_{1}^{1, q}=H^{q+1}\left(M_{2}, M_{1}\right)=0 \quad \text { for } q \neq 0,1, \\
& E_{1}^{2, q}=H^{q+2}\left(M, M_{2}\right)=0 \quad \text { for } q \neq 0,1,2 .
\end{aligned}
$$

Let $G: M \rightarrow \mathbb{R}$ be a Morse-Smale function and let $c_{1}<\cdots<c_{N}$ be its critical points. For $A_{0}<c_{1}<\cdots<c_{N}<A_{N}$ and $K_{i}=G^{-1}\left(\infty, A_{i}\right]$ we get a filtration $K_{0} \subset \cdots \subset K_{N}$. The orientation of $M$ and grad $G$ define an orientation of $L_{a}=G^{-1}(a)$ for each regular value $a$. Giving an orientation to the unstable subspace $E^{u}(x)$ for each critical point $x$ of $G$, and using the orientation of $M$ we also get an orientation of $E^{s}(x)$. Then we have orientations of $W^{u}(x)$ and $W^{s}(x)$. Let $x, y$ be critical points of $G$ of indices $n, n+1$, respectively and let $a$ be a regular value with $G(x)<a<G(y)$. Then $S^{u}(y)=$ $W^{u}(y) \cap L_{a}$ and $S^{s}(x)=W^{s}(x) \cap L_{a}$ are oriented transverse submanifolds of $L_{a}$ with dimensions $n$ and $2-n$, respectively. Therefore, $S^{s}(x) \cap S^{u}(y)$ is a finite set. For each $q \in S^{s}(x) \cap S^{u}(y)$ denote by $I_{q}$ the intersection number.

The proof given by Floer in [2] for the untwisted version of the following lemma can be readily adapted. The only new ingredients are the matrices $\rho(\alpha)$ for nonclosed paths $\alpha$ used to define cohomology with local coefficients.

LEMMA 1. Let $c_{i}, c_{i+1}$ be critical points of $G$ with indices $n, n+1$. For each $q \in$ $S(i, j)=S^{s}\left(c_{i}\right) \cap S^{u}\left(c_{j}\right)$, let $\alpha_{q}(t)=\phi_{\cot (\pi t)}(q): t \in[0,1]$. The coboundary map d: $H^{n}\left(K_{i}, K_{i-1}\right) \rightarrow H^{n+1}\left(K_{i+1}, K_{i}\right)$ is given by 


$$
d=\sum_{q \in S(i, j)} I_{q} \rho\left(\alpha_{q}\right)
$$

To change the Bott integral in a neighborhood of each critical level set, we will use the following propositions:

Proposition 5. Let $F: M \rightarrow \mathbb{R}$ be a Bott function and let $\gamma$ be a critical circle of index $n$. Given a small neighborhood $U$ of $\gamma$ there is another Bott function $G$ which agrees with $F$ outside $U$ and has nondegenerate critical points $w, z \in \gamma$ of indices $n, n+1$ and no other critical points in $U$.

Proof. Let $F(\gamma)=c$. If $\Delta(\gamma)=1$, there is a tubular neighborhood $U$ of $\gamma$ with coordinates $(x, y) \in B_{\varepsilon}(0), \theta \in \times S^{1}=\mathbb{R} / \mathbb{Z}$ such that

$$
F(x, y, \theta)=c+ \pm x^{2} \pm y^{2} .
$$

Let $\rho: \mathbb{R} \rightarrow[0,1]$ be a smooth function with $\rho(t)=0$ if $t>\varepsilon^{2}$ and $\rho(t)=1$ for $t<\varepsilon^{2} / 4$. Define

$$
G= \begin{cases}f+\delta \rho\left(x^{2}+y^{2}\right) \cos \theta, & \text { on } U, \\ f, & \text { outside } U\end{cases}
$$

On $U$ we have

$$
\begin{aligned}
\operatorname{grad} G(x, y, \theta)= & 2\left( \pm 1+\delta \rho^{\prime}\left(x^{2}+y^{2}\right) \cos \theta\right) x \frac{\partial}{\partial x} \\
& +2\left( \pm 1+\delta \rho^{\prime}\left(x^{2}+y^{2}\right) \cos \theta\right) y \frac{\partial}{\partial y} \\
& -\delta \rho\left(x^{2}+y^{2}\right) \sin \theta \frac{\partial}{\partial \theta} .
\end{aligned}
$$

Thus, if $\delta$ is sufficiently small, the only critical points of $G$ in $U$ are $w=(0,0, \pi)$ and $z=(0,0,0)$ with indices $p$ and $p+1$, respectively.

Proposition 6. Let $F: M \rightarrow \mathbb{R}$ be a Bott function and let $\mathbb{T}$ be a minimal (maximal) torus. Given a small neighborhood $U$ of $\mathbb{T}$ there is another Bott function $G$ which

(1) agrees with $F$ outside $U$;

(2) has nondegenerate critical points $p, q, r, s \in \mathbb{T}$ of indices $0,1,1,2$ (or 1,2,2,3) and no other critical points in $U$;

(3) there are no gradient lines either from a critical point of index 1 to any of $q, r, s$ or from a critical point of index 2 to $s$ (either from $p$ to a critical point of index 1 or from $p, q, r, s$ to a critical point of index 2 ).

Proof. Consider the case of a minimal torus. Let $F(\mathbb{T})=c$. There is a tubular neighborhood $U$ of $\mathbb{T}$ with coordinates $(x, \theta, \varphi) \in(-\varepsilon, \varepsilon) \times S^{1} \times S^{1}$ such that $F(x, \theta, \varphi)=$ $c+x^{2}$.

For each critical point $z$ of $F$ of index $2, W^{u}(z) \cap\{x\} \times S^{1} \times S^{1}$ is a curve. For each critical point $w$ of $F$ of index $1, W^{u}(w) \cap\{x\} \times S^{1} \times S^{1}$ is a point. Therefore, we can choose the coordinates $\theta, \varphi$ such that

(a) $(x, 0,0) \notin \bigcup_{u(z)=2} W^{u}(z)$;

(b) $\{x\} \times\left(S^{1}-\{\pi\}\right) \times\{0\} \cap \bigcup_{u(z)=1} W^{u}(z)=\emptyset$;

(c) $\{x\} \times\{0\} \times\left(S^{1}-\{\pi\}\right) \cap \bigcup_{i(w)=2} W^{u}(w)=\emptyset$. 
Let $\rho$ be as in the proof of Proposition 5. Define

$$
G= \begin{cases}F+\rho\left(x^{2}\right)\left(\delta_{1} \cos \theta+\delta_{2} \cos \varphi\right), & \text { on } U, \\ F, & \text { outside } U .\end{cases}
$$

On $U$ we have

$$
\begin{aligned}
\operatorname{grad} G(x, \theta, \varphi)= & 2\left( \pm 1+\rho^{\prime}\left(x^{2}\right)\left(\delta_{1} \cos \theta+\delta_{2} \cos \varphi\right)\right) x \frac{\partial}{\partial x} \\
& -\rho\left(x^{2}\right)\left(\delta_{1} \sin \theta \frac{\partial}{\partial \theta}+\delta_{2} \sin \varphi \frac{\partial}{\partial \varphi}\right) .
\end{aligned}
$$

Thus, if $\delta_{1}, \delta_{2}$ are sufficiently small, the only critical points of $G$ in $U$ are $p=(0, \pi, \pi)$, $q=(0, \pi, 0), r=(0,0, \pi), s=(0,0,0)$ which have the required indices and

(a) $W^{s}(s) \cap U=(-\varepsilon, \varepsilon) \times\{(0,0)\}$;

(b) $W^{s}(q) \cap U=(-\varepsilon, \varepsilon) \times\left(S^{1}-\{\pi\}\right) \times\{0\}$;

(c) $W^{s}(r) \cap U=(-\varepsilon, \varepsilon) \times\{0\} \times\left(S^{1}-\{\pi\}\right)$.

The proof of the maximal torus case is similar.

Applying Proposition 5 to $f$ we obtain a Bott function without critical circles, then using Proposition 6 we obtain a Bott function without critical circles and minimal torus (Klein bottles), and with no gradient lines from a critical point of index $i$ to a critical point of index $j \geq i$. Using Proposition 6 again we finally get a Morse-Smale function $g$ that agrees with $f$ outside a neighborhood of each critical set. Denote by $C_{j}(g)$ the set of critical points of $g$ with index $j$. We have

$$
\begin{aligned}
& C_{0}(g)=\left\{w_{1}, \ldots, w_{k_{1}}, p_{k_{1}+1}, \ldots, p_{k_{2}}\right\}, \\
& C_{1}(g)=\left\{z_{1}, \ldots, z_{k_{1}}, q_{k_{1}+1}, r_{k_{1}+1}, \ldots, q_{k_{2}}, r_{k_{2}}, w_{k_{2}+1}, \ldots, w_{k_{3}}, p_{k_{3}+1}, \ldots, p_{k_{4}}\right\}, \\
& C_{2}(g)=\left\{s_{k_{1}+1}, \ldots, s_{k_{2}}, z_{k_{2}+1}, \ldots, z_{k_{3}}, q_{k_{3}+1}, r_{k_{3}+1}, \ldots, q_{k_{4}}, r_{k_{4}}, w_{k_{4}+1}, \ldots, w_{l}\right\}, \\
& C_{3}(g)=\left\{s_{k_{3}+1}, \ldots, s_{k_{4}}, z_{k_{4}+1}, \ldots, z_{l}\right\} .
\end{aligned}
$$

Proof OF Proposition 3. For $k_{2 n}<i \leq k_{2 n+1}$ let $g\left(w_{i}\right)<d_{i}<g\left(z_{i}\right)$ and $N_{i}^{*}=$ $g^{-1}\left(\infty, d_{i}\right]$. Then, $H^{n}\left(N_{i}^{*}, N_{i-1}\right)=\mathbb{C}^{m} w_{i}, H^{n+1}\left(N_{i}, N_{i}^{*}\right)=\mathbb{C}^{m} z_{i}$, and according to Milnor [7],

$$
\tau_{d\left(N_{i}^{*}, N_{i-1}\right)}=1, \quad \tau_{d\left(N_{i}, N_{i}^{*}\right)}=1 .
$$

Using Lemma 1 we have the sequence

$$
0 \longrightarrow H^{n}\left(N_{i}, N_{i-1}\right) \stackrel{\mathscr{A}_{i}}{\longrightarrow} \mathbb{C}^{m} \stackrel{\mathscr{D}_{i}}{\longrightarrow} \mathbb{C}^{m} \stackrel{\mathscr{B}_{i}}{\longrightarrow} H^{n+1}\left(N_{i}, N_{i-1}\right) \longrightarrow 0
$$

with $\mathscr{D}_{i}$ defined in the the statement of Proposition 3. Therefore,

$$
H^{n}\left(N_{i}, N_{i-1}\right)=\operatorname{ker} \mathscr{D}_{i}, \quad H^{n+1}\left(N_{i}, N_{i-1}\right)=\operatorname{coker} \mathscr{D}_{i} .
$$

Since sequence (3.20) has torsion $\tau\left(\mathscr{D}_{i}\right)^{(-1)^{n}}$, we obtain by Corollary 2

$$
\tau_{0}^{n, i}=\tau_{d\left(N_{i}, N_{i}^{*}\right)} \tau_{d\left(N_{i}^{*}, N_{i-1}\right)} \boldsymbol{\tau}\left(\mathscr{D}_{i}\right)^{(-1)^{n}}=\boldsymbol{\tau}\left(\mathscr{D}_{i}\right)^{(-1)^{n}} .
$$

For $k_{2 n-1}<i \leq k_{2 n}$, let $g\left(p_{i}\right)<c_{i}^{\prime}<g\left(q_{i}\right), g\left(r_{i}\right)<c_{i}^{\prime \prime}<g\left(s_{i}\right)$ and $N_{i}^{\prime}=g^{-1}\left(\infty, c_{i}^{\prime}\right]$, 
$N_{i}^{\prime \prime}=g^{-1}\left(\infty, c_{i}^{\prime \prime}\right]$. Then, $H^{n-1}\left(N_{i}^{\prime}, N_{i-1}\right)=\mathbb{C}^{m} p_{i}, H^{n-1}\left(N_{i}^{\prime \prime}, N_{i}^{\prime}\right)=\mathbb{C}^{m} q_{i} \oplus \mathbb{C}^{m} r_{i}$, $H^{n+1}\left(N_{i}, N_{i}^{\prime \prime}\right)=\mathbb{C}^{m} s_{i}$. Again from [7],

$$
\tau_{d\left(N_{i}^{\prime}, N_{i-1}\right)}=1, \quad \tau_{d\left(N_{i}^{\prime \prime}, N_{i}^{\prime}\right)}=1, \quad \tau_{d\left(N_{i}, N_{i}^{\prime \prime}\right)}=1 .
$$

By Lemma 1 we have the sequence of the triad $\left(N_{i}^{\prime \prime}, N_{i}^{\prime}, N_{i-1}\right)$

$$
0 \longrightarrow H^{n-1}\left(N_{i}^{\prime \prime}, N_{i-1}\right) \stackrel{\mathscr{A}_{i}}{\longrightarrow} \mathbb{C}^{m} \stackrel{\mathscr{D}_{i}}{\longrightarrow} \mathbb{C}^{m} \oplus \mathbb{C}^{m} \stackrel{\mathscr{B}_{i}}{\longrightarrow} H^{n}\left(N_{i}^{\prime \prime}, N_{i-1}\right) \longrightarrow 0,
$$

and the exact sequence of the triad $\left(N_{i}, N_{i}^{\prime \prime}, N_{i}^{\prime}\right)$

$$
0 \longrightarrow H^{1}\left(N_{i}, N_{i}^{\prime}\right) \stackrel{\mathscr{A}_{i}}{\longrightarrow} \mathbb{C}^{m} \oplus \mathbb{C}^{m} \stackrel{\mathscr{I}_{i}^{*}}{\longrightarrow} \mathbb{C}^{m} \stackrel{\mathscr{B}_{i}}{\longrightarrow} H^{2}\left(N_{i}, N_{i}^{\prime}\right) \longrightarrow 0,
$$

with $\mathscr{D}_{i}$ and $\mathscr{D}_{i}^{*}$ defined in the statement of Proposition 3. The first part of the sequence of the triad $\left(N_{i}, N_{i}^{\prime \prime}, N_{i-1}\right)$

$$
0 \longrightarrow H^{n-1}\left(N_{i}, N_{i-1}\right) \longrightarrow H^{n-1}\left(N_{i}^{\prime \prime}, N_{i-1}\right) \longrightarrow 0,
$$

and sequence (3.24) give

$$
\begin{aligned}
H^{n-1}\left(N_{i}, N_{i-1}\right) & =H^{n-1}\left(N_{i}^{\prime \prime}, N_{i-1}\right)=\operatorname{ker} \mathscr{D}_{i}, \\
H^{n}\left(N_{i}^{\prime \prime}, N_{i-1}\right) & =\text { coker } \mathscr{D}_{i} .
\end{aligned}
$$

Using (3.24) and (3.25), we have the commutative diagram

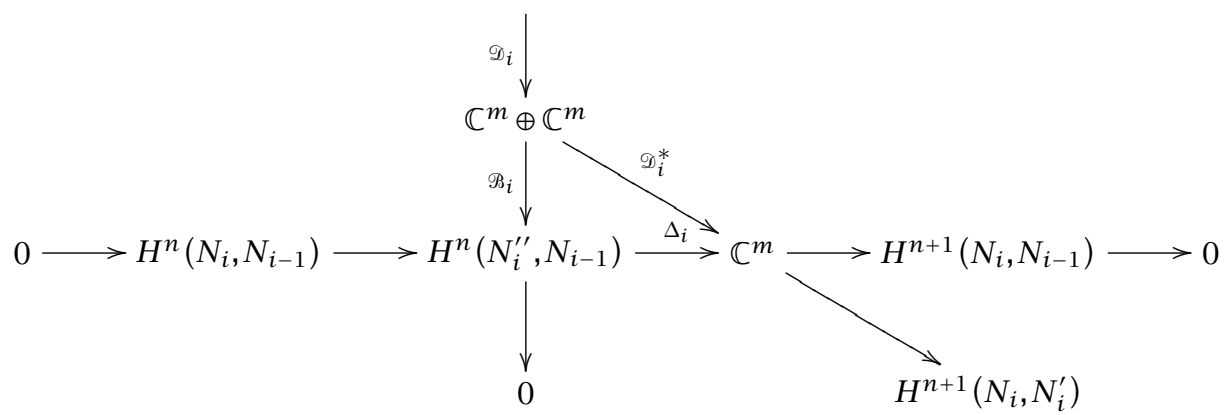

which implies

$$
\begin{aligned}
H^{n}\left(N_{i}, N_{i-1}\right) & =\operatorname{ker} \Delta_{i}=\mathscr{P}_{i}\left(\operatorname{coker} \mathscr{D}_{i} \cap \operatorname{ker} \mathscr{D}_{i}^{*}\right), \\
H^{n+1}\left(N_{i}, N_{i-1}\right) & =\operatorname{coker} \Delta_{i}=\operatorname{coker} \mathscr{D}_{i}^{*}=H^{n+1}\left(N_{i}, N_{i}^{\prime}\right) .
\end{aligned}
$$

Sequence (3.24) has torsion $\tau\left(\mathscr{D}_{i}\right)^{(-1)^{n-1}}$ and sequence (3.25) has torsion $\tau\left(\Delta_{i}\right)^{(-1)^{n-1}}$. Therefore, Corollary 2 gives

$$
\begin{aligned}
\tau_{d\left(N_{i}^{\prime \prime}, N_{i-1}\right)} & =\tau_{d\left(N_{i}^{\prime \prime}, N_{i}^{\prime}\right)} \boldsymbol{\tau}_{d\left(N_{i}^{\prime}, N_{i-1}\right)} \boldsymbol{\tau}\left(\mathscr{D}_{i}\right)^{(-1)^{n}}=\boldsymbol{\tau}\left(\mathscr{D}_{i}\right)^{(-1)^{n}}, \\
\boldsymbol{\tau}_{d_{0}^{n-1, i}} & =\boldsymbol{\tau}_{d\left(N_{i}, N_{i}^{\prime \prime}\right)} \boldsymbol{\tau}_{d\left(N_{i}^{\prime \prime}, N_{i-1}\right)} \boldsymbol{\tau}\left(\Delta_{i}\right)^{(-1)^{n-1}}=\boldsymbol{\tau}\left(\mathscr{D}_{i}\right)^{(-1)^{n}} \boldsymbol{\tau}\left(\Delta_{i}\right)^{(-1)^{n-1} .} .
\end{aligned}
$$

To compute $\tau\left(\mathscr{D}_{i}\right)$, we recall that $\mathscr{D}_{i}=\left(\begin{array}{c}I-\rho\left(\alpha_{i}\right) \\ I \pm \rho\left(\beta_{i}\right)\end{array}\right)$. Since $\rho\left(\alpha_{i}\right), \rho\left(\beta_{i}\right) \in U(m)$ and they commute. There is a splitting

$$
\mathbb{C}^{m}=V_{\alpha_{i}}+V_{\beta_{i}}+\operatorname{ker} \mathscr{D}_{i},
$$

invariant under $\rho\left(\alpha_{i}\right)$, and $\rho\left(\beta_{i}\right)$, such that $I-\rho\left(\alpha_{i}\right): V_{\alpha_{i}} \hookleftarrow$ and $I-\rho\left(\beta_{i}\right): V_{\beta_{i}} \hookleftarrow$ are 
nonsingular. Let $\mathbf{k}_{i}, \mathbf{v}_{\alpha_{i}}, \mathbf{v}_{\beta_{i}}$ be bases of $H^{n-1}\left(N_{i}, N_{i-1}\right)=\operatorname{ker} \mathscr{D}_{i}, V_{\alpha_{i}}, V_{\beta_{i}}$. Let

$$
W_{i}=\left(V_{\beta_{i}}+\operatorname{ker} \mathscr{D}_{i}\right) \oplus\left(V_{\alpha_{i}}+\operatorname{ker} \mathscr{D}_{i}\right),
$$

then $j: W_{i} \rightarrow H^{n}\left(N_{i}^{\prime \prime}, N_{i-1}\right)$ is an isomorphism, and so $j\left[\left(\mathbf{v}_{\beta_{i}} \cup \mathbf{k}_{i}\right) \times\{0\}\right] \cup j[\{0\} \times$ $\left.\left(\mathbf{v}_{\alpha_{i}} \cup \mathbf{k}_{i}\right)\right]$ is a basis of $H^{n}\left(N_{i}^{\prime \prime}, N_{i-1}\right)$. Thus,

$$
\begin{aligned}
\tau\left(\mathscr{D}_{i}\right)= & \frac{\wedge\left(\mathscr{D}_{i} \mathbf{v}_{\alpha_{i}}, \mathscr{D}_{i} \mathbf{v}_{\beta_{i}},\left(\mathbf{v}_{\beta_{i}} \cup \mathbf{k}_{i}\right) \times\{0\},\{0\} \times\left(\mathbf{v}_{\alpha_{i}} \cup \mathbf{k}_{i}\right)\right) \otimes \wedge \mathbf{k}_{i}}{\wedge\left(\mathbf{v}_{\alpha_{i}}, \mathbf{v}_{\beta_{i}}, \mathbf{k}_{i}\right) \otimes \wedge\left(\mathscr{B}_{i}\left[\left(\mathbf{v}_{\beta_{i}} \cup \mathbf{k}_{i}\right) \times\{0\}\right], \mathscr{B}_{i}\left[\{0\} \times\left(\mathbf{v}_{\alpha_{i}} \cup \mathbf{k}_{i}\right)\right]\right)} \\
= & \frac{\wedge\left(\left(I-\rho\left(\alpha_{i}\right)\right) \mathbf{v}_{\alpha_{i}}, \mathbf{v}_{\beta_{i}}, \mathbf{k}_{i}\right) \otimes \wedge\left(\mathbf{v}_{\alpha_{i}},\left(I-\rho\left(\beta_{i}\right)\right) \mathbf{v}_{\beta_{i}}, \mathbf{k}_{i}\right) \otimes \wedge \mathbf{k}_{i}}{\wedge\left(\mathbf{v}_{\alpha_{i}}, \mathbf{v}_{\beta_{i}}, \mathbf{k}_{i}\right) \otimes \mathscr{B}_{i}^{*}\left(\wedge\left(\mathbf{v}_{\beta_{i}}, \mathbf{k}_{i}\right) \otimes \wedge\left(\mathbf{v}_{\alpha_{i}}, \mathbf{k}_{i}\right)\right)} .
\end{aligned}
$$

To compute $\tau\left(\Delta_{i}\right)$ we recall that $\mathscr{D}_{i}^{*}=\left(I \pm \rho\left(\beta_{i}\right), \rho\left(\alpha_{i}\right)-I\right)$ and then

$$
W_{i} \cap \operatorname{ker} \mathscr{D}_{i}^{*}=\operatorname{ker} \mathscr{D}_{i} \oplus \operatorname{ker} \mathscr{D}_{i}, \quad \mathscr{D}_{i}^{*}\left(\mathbb{C}^{m} \oplus \mathbb{C}^{m}\right)=\mathscr{D}_{i}^{*}\left(W_{i}\right)=V_{\beta_{i}}+V_{\alpha_{i}} .
$$

Thus, $\operatorname{ker} \Delta_{i}=\mathscr{B}_{i}\left(\operatorname{ker} \mathscr{D}_{i} \oplus \operatorname{ker} \mathscr{D}_{i}\right)$ and coker $\Delta_{i}=\operatorname{coker} \mathscr{D}_{i}^{*}=\operatorname{ker} \mathscr{D}_{i}$. Thus, $H^{n}\left(N_{i}, N_{i-1}\right)$ $\cong \operatorname{ker} \mathscr{D}_{i} \oplus \operatorname{ker} \mathscr{D}_{i}, H^{n}\left(N_{i}, N_{i-1}\right)=\operatorname{ker} \mathscr{D}_{i}$, and using $\mathbf{k}_{i} \times \mathbf{k}_{i}$ and $\mathbf{k}_{i}$ as their bases we have

$$
\boldsymbol{\tau}\left(\Delta_{i}\right)=\frac{\wedge\left(\left(I-\rho\left(\alpha_{i}\right)\right) \mathbf{v}_{\alpha_{i}},\left(I-\rho\left(\beta_{i}\right)\right) \mathbf{v}_{\beta_{i}}, \mathbf{k}_{i}\right) \otimes\left(\wedge \mathbf{k}_{i} \otimes \wedge \mathbf{k}_{i}\right)}{\mathscr{R}_{i}^{*}\left(\wedge\left(\mathbf{v}_{\beta_{i}}, \mathbf{k}_{i}\right) \otimes \wedge\left(\mathbf{v}_{\alpha_{i}}, \mathbf{k}_{i}\right)\right) \otimes \wedge \mathbf{k}_{i}} .
$$

From equations (3.31), (3.34), and (3.36) we have

$$
\tau_{d_{0}^{n-1, i}}=1 .
$$

We now come to the description of the components $F_{i j}^{*}$ of $d_{1}$. Let $\psi_{t}$ be the gradient flow of $g$. One can construct an index filtration

$$
\emptyset=K_{-1} \subset K_{0} \subset L_{1} \subset P_{1} \subset K_{1} \subset L_{2} \subset P_{2} \subset K_{2} \subset K_{3}=M,
$$

such that $L_{1} \subset M_{1} \subset L_{2}, P_{1} \subset M_{2} \subset P_{2}$ and

$$
\begin{aligned}
& \bigcap_{t \in \mathbb{R}} \psi_{t}\left(K_{i} \backslash K_{i-1}\right)=C_{i}(g), \quad i=0,1,2,3, \\
& \bigcap_{t \in \mathbb{R}} \psi_{t}\left(L_{i} \backslash K_{i-1}\right)=C_{i}(g) \cap M_{1}, \quad i=1,2, \\
& \bigcap_{t \in \mathbb{R}} \psi_{t}\left(P_{i} \backslash L_{i}\right)=C_{i}(g) \cap\left(M_{2} \backslash M_{1}\right), \quad i=1,2, \\
& \bigcap_{t \in \mathbb{R}} \psi_{t}\left(K_{i} \backslash P_{i}\right)=C_{i}(g) \backslash M_{2}, \quad i=1,2 .
\end{aligned}
$$

We have

$$
H^{i}\left(K_{i}, K_{i-1}\right)=\bigoplus_{\operatorname{index~}(x)=i} \mathbb{C}^{m} x .
$$

Note that all the components $G_{x y}^{*}: \mathbb{C}^{m} x \rightarrow \mathbb{C}^{m} y$ of the maps $d^{K}$ in the cochain complex

$$
0 \longrightarrow H^{0}\left(K_{0}\right) \stackrel{d^{K}}{\longrightarrow} H^{1}\left(K_{1}, K_{0}\right) \stackrel{d^{K}}{\longrightarrow} H^{2}\left(K_{2}, K_{1}\right) \stackrel{d^{K}}{\longrightarrow} H^{2}\left(M, K_{2}\right) \longrightarrow 0
$$


are given as in equation (3.12) of Lemma 1.

THEOREM 1. For critical circles $\gamma_{i}$ the components $F_{i j}^{k}$ are induced by the maps

- (c.c) $G_{w_{i} w_{j}}^{n}, G_{z_{i} z_{j}}^{n+1}$ for $k_{2 n}<i \leq k_{2 n+1}, k_{2 n+2}<j \leq k_{2 n+3}, n=0,1$,

- (c.t) $G_{w_{i} q_{j}}^{1}, G_{w_{i} r_{j}}^{1}, G_{z_{i} s_{j}}^{2}$ for $k_{2}<i \leq k_{3}<j \leq k_{4}$.

For critical tori $F_{c_{i}}$ the components $F_{i j}^{k}$ are induced by the maps

- (t.c) $G_{p_{i} w_{j}}^{0}, G_{q_{i} z_{j}}^{1}, G_{r_{i} z_{j}}^{1}$ for $k_{1}<i \leq k_{2}<j \leq k_{3}$.

Proof. Consider the commutative diagrams

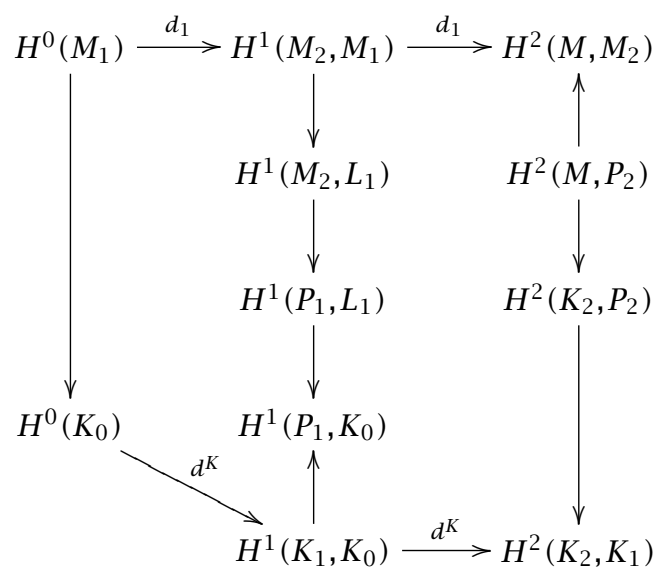

and

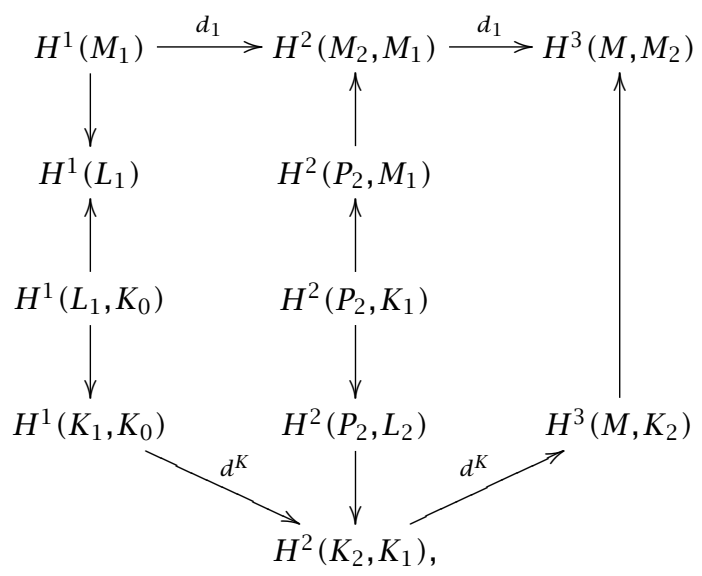

where the downwards maps are injective and the upwards maps are surjective. By (3.42) the maps $d_{1}$ in

$$
H^{0}\left(M_{1}\right) \stackrel{d_{1}}{\longrightarrow} H^{1}\left(M_{2}, M_{1}\right) \stackrel{d_{1}}{\longrightarrow} H^{2}\left(M, M_{2}\right)
$$

are induced by the components $H^{0}\left(K_{0}\right) \rightarrow H^{1}\left(P_{1}, L_{1}\right)$ and

$$
H^{1}\left(P_{1}, L_{1}\right)=\bigoplus_{k_{2}<i \leq k_{3}} \mathbb{C}^{m} w_{i} \longrightarrow H^{2}\left(K_{2}, P_{2}\right)=\bigoplus_{k_{4}<i} \mathbb{C}^{m} w_{i} \oplus \bigoplus_{k_{3}<i \leq k_{4}} \mathbb{C}^{m} q_{i} \oplus \mathbb{C}^{m} r_{i}
$$


of the maps $d^{K}$ in (3.41). By (3.43) the maps $d_{1}$ in

$$
H^{1}\left(M_{1}\right) \stackrel{d_{1}}{\longrightarrow} H^{2}\left(M_{2}, M_{1}\right) \stackrel{d_{1}}{\longrightarrow} H^{3}\left(M, M_{2}\right)
$$

are induced by the components

$$
H^{1}\left(L_{1}, K_{0}\right)=\bigoplus_{i \leq k_{1}} \mathbb{C}^{m} z_{i} \oplus \bigoplus_{k_{1}<i \leq k_{2}} \mathbb{C}^{m} q_{i} \oplus \mathbb{C}^{m} r_{i} \longrightarrow H^{2}\left(P_{2}, L_{2}\right)=\bigoplus_{k_{2}<i \leq k_{3}} \mathbb{C}^{m} z_{i}
$$

and $H^{2}\left(P_{2}, L_{2}\right) \rightarrow H^{3}\left(M, K_{2}\right)$ of the maps $d^{K}$ in (3.41).

We now consider the term $\left(E_{2}, d_{2}\right)$ of the spectral sequence defined by the filtration. By (2.12) and Corollary 4 , the spaces $E_{2}^{n, q}$ that can be nonzero are

$$
\begin{aligned}
E_{2}^{0, q} & =\operatorname{ker}\left(d_{1}: H^{q}\left(M_{1}\right) \rightarrow H^{q+1}\left(M_{2}, M_{1}\right)\right), \quad q=0,1, \\
E_{2}^{0,2} & =H^{2}\left(M_{1}\right), \\
E_{2}^{1, q} & =\frac{\operatorname{ker}\left(d_{1}: H^{q+1}\left(M_{2}, M_{1}\right) \longrightarrow H^{q+2}\left(M, M_{2}\right)\right)}{d_{1}\left(H^{q}\left(M_{1}\right)\right)}, \quad q=0,1, \\
E_{2}^{2,-1} & =H^{1}\left(M, M_{2}\right), \\
E_{2}^{2, q} & =\frac{H^{q+2}\left(M, M_{2}\right)}{d_{1}\left(H^{q+1}\left(M_{2}, M_{1}\right)\right)}, \quad q=0,1,
\end{aligned}
$$

Therefore, we can have nonzero maps $d_{2}: E_{2}^{n, q} \rightarrow E_{2}^{n+2, q-1}$ only for $n=0, q=0,1,2$.

THEOREM 2. (1) The map $d_{2}: E_{2}^{0,0} \rightarrow E_{2}^{2,-1}$ is induced by the component

$$
H^{0}\left(K_{0}\right) \longrightarrow \bigoplus_{k_{3}<i \leq k_{4}} \mathbb{C}^{m} p_{i} \text { of } d^{K}: H^{0}\left(K_{0}\right) \longrightarrow H^{1}\left(K_{1}, K_{0}\right)
$$

(2) The $\operatorname{map} d_{2}: E_{2}^{0,1} \rightarrow E_{2}^{2,0}$ is induced by the component

$$
\bigoplus_{i \leq k_{1}} \mathbb{C}^{m} z_{i} \oplus \bigoplus_{k_{1}<i \leq k_{2}} \mathbb{C}^{m} q_{i} \oplus \mathbb{C}^{m} r_{i} \longrightarrow \bigoplus_{k_{4}<i} \mathbb{C}^{m} w_{i} \oplus \bigoplus_{k_{3}<i \leq k_{4}} \mathbb{C}^{m} q_{i} \oplus \mathbb{C}^{m} r_{i}
$$

of $d^{K}: H^{1}\left(K_{1}, K_{0}\right) \rightarrow H^{2}\left(K_{2}, K_{1}\right)$.

(3) The $\operatorname{map}_{2}: E_{2}^{0,2} \rightarrow E_{2}^{2,1}$ is induced by the component

$$
\bigoplus_{k_{1}<i \leq k_{2}} \mathbb{C}^{m} s_{i} \longrightarrow H^{3}\left(M, K_{2}\right) \text { of } d^{K}: H^{2}\left(K_{2}, K_{1}\right) \longrightarrow H^{3}\left(M, K_{2}\right)
$$

Proof. By (2.14), the map $d_{2}: E_{2}^{0, q} \rightarrow E_{2}^{2, q-1}$ is given as the composition

$$
J_{q}^{-1} \circ \delta_{q} \mid \operatorname{im} k_{q}: \operatorname{im} k_{q} \longrightarrow \frac{H^{q+1}\left(M, M_{2}\right)}{\operatorname{im} j_{q}},
$$

where $k_{q}: H^{q}\left(M_{2}\right) \rightarrow H\left(M_{1}\right), \delta: H^{q}\left(M_{1}\right) \rightarrow H^{q+1}\left(M, M_{1}\right)$ is the coboundary map and $j_{q}: H^{q+1}\left(M, M_{2}\right) \rightarrow H^{q+1}\left(M, M_{1}\right)$ defines the isomorphism $J_{q}: H^{q+1}\left(M, M_{2}\right) / \operatorname{ker} j_{q} \rightarrow$ 
$\operatorname{im} j_{q}$. Consider the commutative diagrams

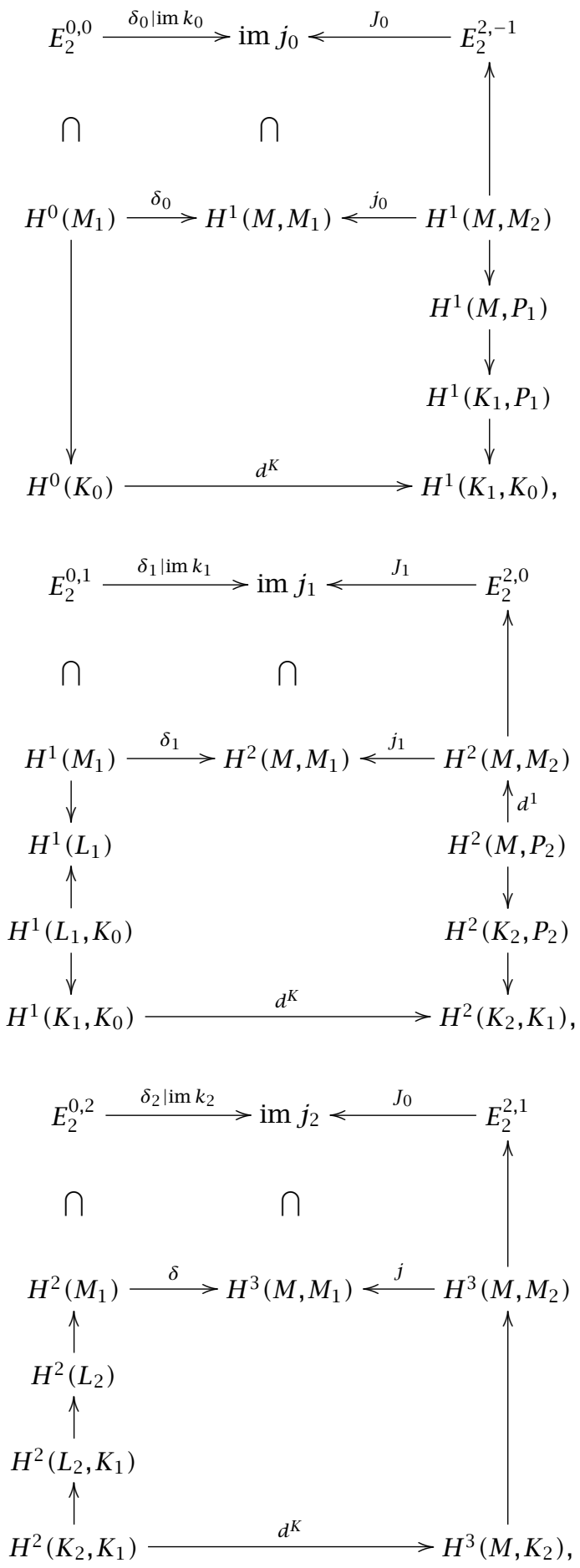


where as in Theorem 1 the downwards maps are injective and the upwards maps are surjective. By (3.53) and $H^{1}\left(K_{1}, P_{1}\right)=\bigoplus_{k_{3}<i \leq k_{4}} \mathbb{C}^{m} p_{i}$, we get (1). By (3.54), $H^{1}\left(L_{1}, K_{0}\right)=$ $\bigoplus_{i \leq k_{1}} \mathbb{C}^{m} z_{i} \oplus \bigoplus_{k_{1}<i \leq k_{2}} \mathbb{C}^{m} q_{i} \oplus \mathbb{C}^{m} r_{i}$ and $H^{2}\left(K_{2}, P_{2}\right)=\bigoplus_{k_{4}<i} \mathbb{C}^{m} w_{i} \oplus \bigoplus_{k_{3}<i \leq k_{4}} \mathbb{C}^{m} q_{i} \oplus$ $\mathbb{C}^{m} r_{i}$ we get (2). By (3.54) and $H^{2}\left(L_{2}, K_{1}\right)=\bigoplus_{k_{1}<i \leq k_{2}} \mathbb{C}^{m} s_{i}$, we get (3).

4. Examples. Consider the following instance of the Kovalevskaya integrable case of the rigid body. There are two minimal circles $m_{1}, m_{2}$, two nonorientable and one orientable saddle circles $r_{1}, r_{2}, r_{3}$, and one maximal circle $n$. The family of tori starting at $m_{i}$ changes to a family $\mathscr{F}_{i}$ of tori when crossing $r_{i}(i=1,2)$. The families $\mathscr{F}_{1}$ and $\mathscr{F}_{2}$ come together to become one family when crossing $r_{3}$. The manifold $M$ is the 3-dimensional real projective space and so $\pi_{1}(M)=\mathbb{Z}_{2}$. A representation $\rho: \pi_{1}(M) \rightarrow$ $U(1)$ is given by $\rho([0])=1, \rho([1])=-1$.

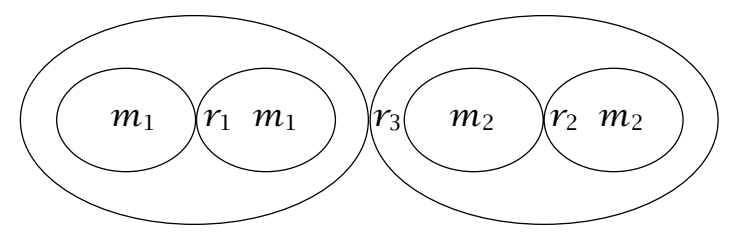

We have that $\rho\left(m_{i}\right)=1, \rho\left(r_{j}\right)=\rho(n)=-1, \Delta\left(r_{i}\right)=-1, \Delta\left(r_{3}\right)=1, i=1,2, j=1,2,3$. Therefore,

$$
\begin{aligned}
& H^{k}\left(M_{1}\right)= \begin{cases}\mathbb{C} \oplus \mathbb{C}, & \text { if } k=0,1, \\
0, & \text { in other case, }\end{cases} \\
& H^{k}\left(M_{2}, M_{1}\right)= \begin{cases}\mathbb{C} \oplus \mathbb{C}, & \text { if } k=1,2, \\
0, & \text { in other case, }\end{cases} \\
& H^{*}\left(M, M_{2}\right)=0 \\
& \tau_{d_{0}^{0, i}}=\tau_{d_{0}^{1, i}}=1 \quad(i=1,2), \quad \tau_{d_{0}^{1,3}}=\frac{1}{2}, \quad \tau_{d_{0}^{2}}=2 \Longrightarrow \tau_{d_{0}}=1 .
\end{aligned}
$$

We now change the Bott integral $f$ to a Morse-Smale function $g$ to be able to compute $d_{1}: H^{k}\left(M_{1}\right) \rightarrow H^{k+1}\left(M_{2}, M_{1}\right), k=0,1$. Note that $W^{u}\left(r_{i}\right), i=1,2$ is a Möbius strip $\Sigma_{i}$ with $\partial \Sigma_{i}=m_{i}$. The function $g$ has critical points $w_{i}, z_{i}$ on $m_{i}$ with indices 0,1 , respectively, and critical points $\eta_{i}, \zeta_{i}$ on $r_{i}$ with indices 1,2 , respectively. There is one orbit $\alpha_{i}$ connecting $\zeta_{i}$ to $z_{i}$ and two orbits $\beta_{i}, \delta_{i}$ connecting $\eta_{i}$ to $w_{i}$, with $\rho\left(\alpha_{i}\right)=1$, $\rho\left(\beta_{i}\right)=1, \rho\left(\delta_{i}\right)=-1$. Thus, $G_{w_{i} \eta_{i}}: \mathbb{C} \rightarrow \mathbb{C}=2$ and $G_{z_{i} \zeta_{i}}: \mathbb{C} \rightarrow \mathbb{C}=1$.

Therefore, $d_{1}: H^{0}\left(M_{1}\right) \rightarrow H^{1}\left(M_{2}, M_{1}\right)=\left(\begin{array}{ll}2 & 0 \\ 0 & 2\end{array}\right)$ and $d_{1}: H^{1}\left(M_{1}\right) \rightarrow H^{2}\left(M_{2}, M_{1}\right)=\left(\begin{array}{ll}1 & 0 \\ 0 & 1\end{array}\right)$. Thus, $E_{2}=0$ and $\tau_{d_{1}}=2 \cdot 2 / 1=4$.

ACKnowledgement. Parts of this article were written while the first author was visiting the Instituto de Matematicas Universidad Nacional Autonoma de Mexico and Erwin Schrödinger International Institute for Mathematical Physics in Wien. The first author is indebted to these institutions for their invitations, support, and hospitality. 


\section{REFERENCES}

[1] A. L. Fel'shtyn, Attractors, integrable Hamiltonian systems and the Reidemeister torsion, Seminar on Dynamical Systems (Basel) (S. Kuksin et al., eds.), Progr. Nonlinear Differential Equations Appl., vol. 12, Birkhäuser, 1994, pp. 227-234. MR 95c:58140. Zbl 797.58047.

[2] A. Floer, Witten's complex and infinite-dimensional Morse theory, J. Differential Geom. 30 (1989), no. 1, 207-221. MR 90d:58029. Zbl 678.58012.

[3] A. T. Fomenko, Symplectic Geometry, Advanced Studies in Contemporary Mathematics, vol. 5, Gordon and Breach Science Publishers, New York, 1988, Translated from Russian by R. S. Wadhwa. MR 90k:58065. Zbl 716.53005.

[4] A. T. Fomenko and H. Zieschang, On the topology of three-dimensional manifolds arising in Hamiltonian mechanics, Soviet Math. Dokl. 35 (1987), no. 3, 529-534, (translation from Dokl. Akad. Nauk SSSR 294 (1987), no. 2, 283-287, MR 88m:57020). Zbl 734.57014 .

[5] D. S. Freed, Reidemeister torsion, spectral sequences, and Brieskorn spheres, J. Reine Angew. Math. 429 (1992), 75-89. MR 93k:57052. Zbl 743.57015.

[6] D. Fried, Lefschetz formulas for flows, The Lefschetz centennial conference, Part III (Providence, RI), Contemp. Math., vol. 58, Amer. Math. Soc., 1987, pp. 19-69. MR 88k:58138. Zbl 619.58034.

[7] J. Milnor, Whitehead torsion, Bull. Amer. Math. Soc. 72 (1966), 358-426. MR 33\#4922. Zbl 147.23104.

[8] J. W. Milnor, Infinite cyclic coverings, Conference on the Topology of Manifolds (Boston), Michigan State Univ., Prindle, Weber \& Schmidt, 1968, pp. 115-133. MR 39\#3497. Zbl 179.52302.

[9] D. B. Ray and I. M. Singer, $R$-torsion and the Laplacian on Riemannian manifolds, Adv. in Math. 7 (1971), 145-210. MR 45 4447. Zbl 239.58014.

[10] K. Reidemeister, Homotopieringe und Linsenraeüme, Abh. Math. Sem. Univ. Hamburg 11 (1935), 102-109. Zbl 011.32404.

[11] H. Sánchez-Morgado, Reidemeister torsion and Morse-Smale flows, Ergodic Theory Dynamical Systems 16 (1996), no. 2, 405-414. MR 97e:58134. Zbl 872.58041.

[12] F. Waldhausen, Eine Klasse von 3-dimensionalen Mannigfaltigkeiten. I, II, Invent. Math 3 (1967), 308-333. MR 38\#3880. Zbl 168.44503.

FEL'SHTYN: INSTITUT FÜR MATHEMATIK, E.-M.-ARNDT-UNIVERSITÄT GREIFSWALD, JAHN-STRASSE 15A, D-17489 GREIFSWALD, GERMANY

E-mail address: fe1shtynarz.uni-greifswald.de

Morgado: Instituto de Mathematicas, Universidad Nacional Autonoma de MeXico, Ciudad Universitaria C. P. 04510, MeXico D. F., MeXico

E-mail address: hector@math.unam.mx 


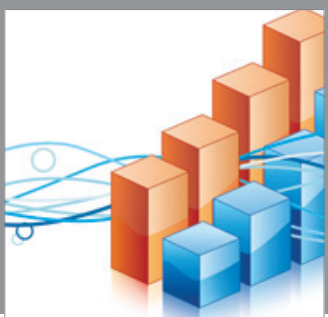

Advances in

Operations Research

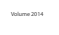

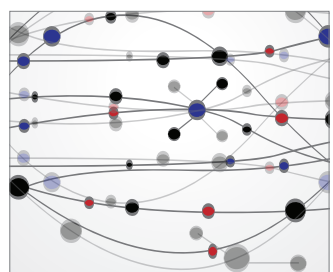

\section{The Scientific} World Journal
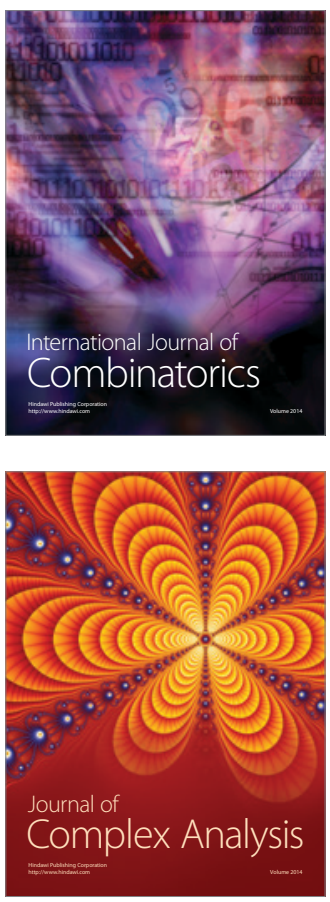

International Journal of

Mathematics and

Mathematical

Sciences
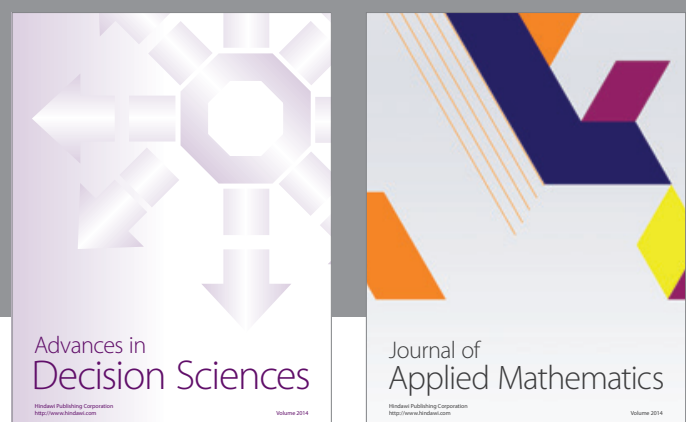

Journal of

Applied Mathematics
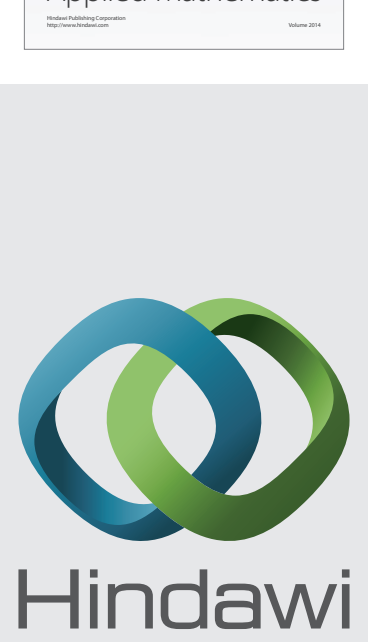

Submit your manuscripts at http://www.hindawi.com
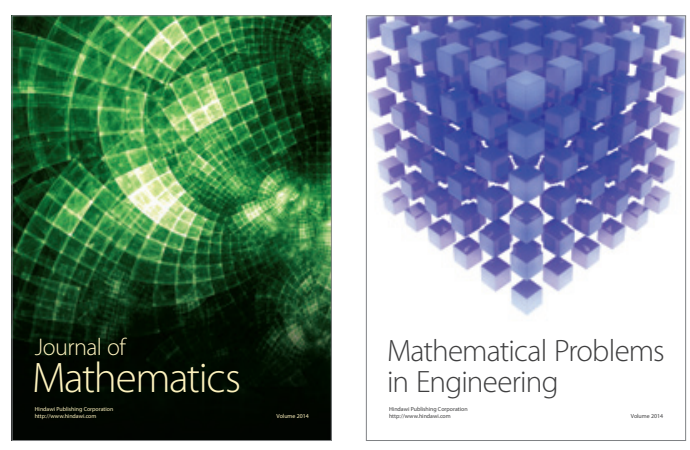

Mathematical Problems in Engineering
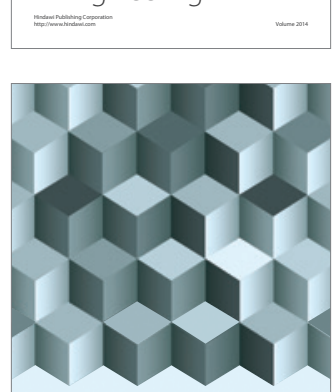

Journal of

Function Spaces
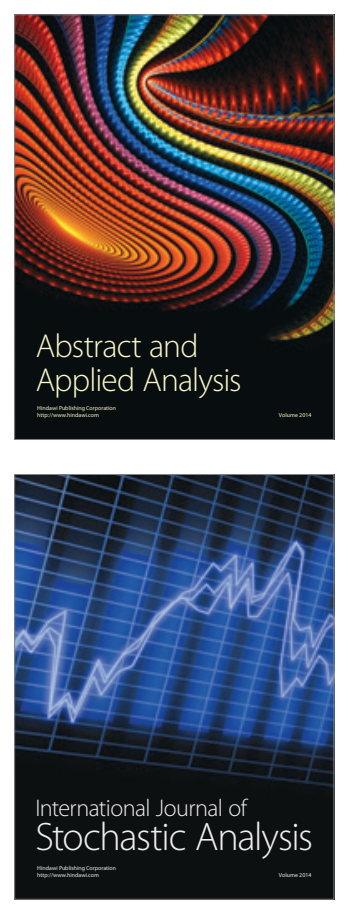

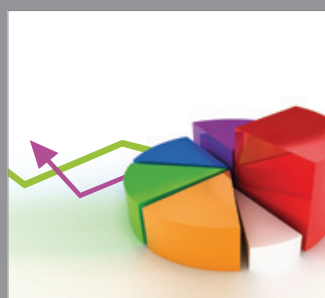

ournal of

Probability and Statistics

Promensencen
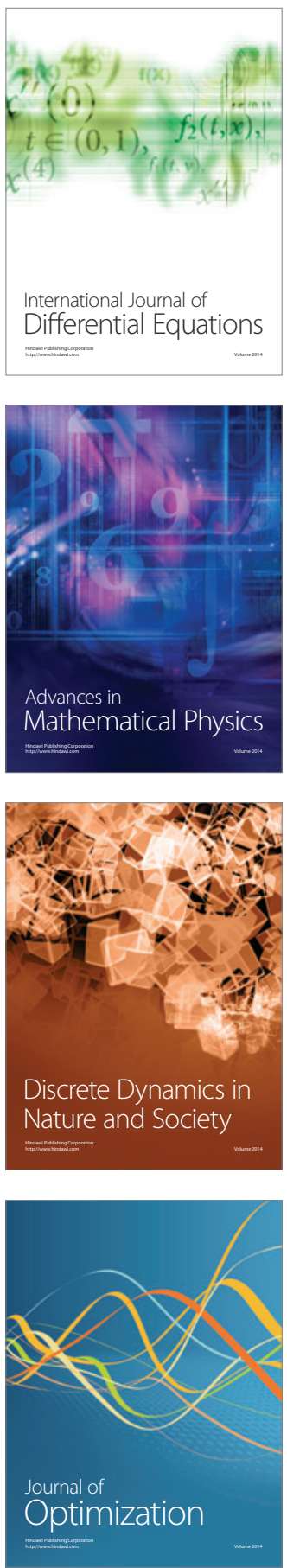